\title{
Das demokratische Potenzial des Internets
}

\author{
Maika Büschenfeldt
}

\section{Zusammenfassung}

Weblogs und komplexe Diskurs-Plattformen repräsentieren ganz unterschiedliche Ausprägungen elektronischer Demokratie. Im Unterschied zu den monolithischen, top-down moderierten Diskurs-Plattformen knüpfen typische Web 2.0 Anwendungen wie Weblogs an die many-to-many-Kommunikationsstruktur des Internets an. Das demokratische Potenzial der Web 2.0 Anwendungen gründet in den Prinzipien der Selbstorganisation aus den frühen Tagen des Internets. Durch Gestaltungsprinzipien wie Offenheit, Einfachheit, bottom up, Nutzerpartizipation, Konsensorientierung und nicht-hierarchischer Organisation werden Web 2.0 Anwendungen zu Manifestationen der Urideen und Konzepte des Cyberspace.

Durch die Fokussierung auf die Interaktion zwischen Software und Nutzern kann die Debatte um die Digitale Spaltung der Gesellschaft eine neue Wendung bekommen: Unter der Annahme, dass Software die Form politischer Auseinandersetzung regelt, liegt das demokratische Potenzial des Webs nicht nur in den Fähigkeiten oder Unfähigkeiten der Nutzer, sondern auch im Design technischer Architekturen.

\section{Einleitung}

Auf der Website des Bundesinnenministeriums wird EDemokratie als Sammelbegriff definiert, der alle Maßnahmen zusammenfasst, »bei denen Internettechnologien eingesetzt werden, um Bürgerinnen und Bürgern zusätzliche demokratische Mitbestimmungs- und Gestaltungsmöglichkeiten einzuräumen.«[1] Diese Definition legt nahe, das Interesse vordringlich auf Angebote der E-Partizipation zu richten, somit auf Verfahren, die darauf abzielen, die Beteiligungsmöglichkeiten der Bürger und Bürgerinnen mit dem Ziel zu erweitern, eine neue Qualität in das Verhältnis von Regierenden und Regierten zu bringen. Im Feld elektronischer Partizipation hat sich vor allem das Konzept »online-moderierter Verfahren« etablieren können. Bürgerbeteiligung wird hier über komplexe Diskursplattformen in moderierten
Abstract

Weblogs and complex designed discourse-platforms represent different types of E-Democracy. In contrast to the monolitic, top-down moderated discourse-platforms typical web 2.0 applications like weblogs are connected to the many-to-many communication structure of the internet. The democratic potencial of web 2.0 applications is based on the principles of self-organisation deriving from the early days of the internet. Web 2.0 applications become manifestations of the origin ideas and concepts of the cyberspace by design principles such as openess, simplicity, bottom up, user participation, consensus and non hierarchical organisation.

Focusing on the interaction between software and users the dispute about the digital divide of society might take a different turn: Assuming that software rules the form of political debates, the web's democratic potencials and risks are not only bound to abilities or inabilities of users but also to the design of technical architectures.
Online-Dialogen umgesetzt [2]. Zum Einsatz kommt dieser Verfahrenstyp in Visions- und Leitbildprozessen, in formellen Verfahren in der Stadtplanung sowie in informellen Verfahren zur Verteilung von Ressourcen, Anlagen und Einrichtungen. E-Partizipation steht hier für eine Beteiligunspraxis, in der das politisch-administrative System den Bürgern den Auftrag erteilt, sich zu vorgegebenen Themen Gedanken zu machen (Märker 2007, S. 254 f).

Mit diesem Verfahrenstypus ist es zwar einerseits gelungen, ein praktikables Konzept der E-Partizipation zu etablieren, andererseits zeigen sich in den komplizierten Verfahren der Entscheidungsfindung sowie der aufwändigen und teuren Qualitäts- und Ergebnissicherung auch die Schwächen dieses Konzeptes. Hinzu kommt, dass die Auftraggeber bislang wenig Willen gezeigt haben, Kontrolle abzugeben, und bürokratische Vorgaben 
wenig Raum für Innovation lassen. Die zur Beteiligung aufgeforderten Bürger haben in der Regel weder Einfluss auf die Wahl der Themen, noch auf die Umsetzung der entwickelten Ideen und Verfahren. Darüber hinaus haben die Ergebnisse der öffentlichen Diskurse weder Rechtswirksamkeit, noch sind diese institutionell verankert [3]. Christoph Dowe, Geschäftsführer des Vereins poldi.net, fasst diese Schwächen pointiert zusammen:

»Keine Website wird veröffentlicht, bevor nicht alle Eventualitäten kalkuliert wurden. Experimente werden nicht gemacht, da sie die Möglichkeit des Scheiterns beinhalten. Ein Modellprojekt kann nur genehmigt werden, wenn es bereits ohne Auffälligkeiten erfolgreich erprobt wurde (und damit kein Modellprojekt mehr ist); ein Modellprojekt darf nur begrenzt neue Wege gehen; ein Modellprojekt darf die Macht der Institutionen nicht in Frage stellen, um sie zu verbessern. Affirmation statt Dialog steht im Vordergrund. Kein guter Nährboden für innovative neue Wege der politischen Kommunikation.« (Dowe 2007, S. 212)

In bürokratischen Vorgaben und der Orientierung an den Verfahren der Offline-Welt mag der Grund liegen, warum sich die innovativeren Beispiele politischer Kommunikation eher jenseits online-moderierter Verfahren und jenseits der offiziellen Förderung durch staatliche Auftraggeber finden lassen. Die visionären Ideen, Techniken und Tools der Online-Kommunikation sind in der Regel nicht speziell für die politische Kommunikation entwickelt worden und haben dennoch in der Diskussion um die demokratischen Potenziale des Internets große Aufmerksamkeit erregt. Das gilt insbesondere für Anwendungen, die dem Themenkreis Web 2.0 zugeordnet werden und ganz besonders für eine, im Vergleich zu den aufwändigen Diskurs-Plattformen, sehr einfache Software, den Weblog. Weblogs (oder kurz Blogs) zählen neben Wikis zu den Personal Publishing Systemen und sind Beispiel für eine dezentrale Struktur der Erzeugung von nutzergenerierten Webinhalten (user generated content). Obwohl der absolute Anteil politischer Blogs an deren Gesamtzahl vergleichsweise gering ist, wird die politische Wirksamkeit der politischen Blogosphäre[4] dennoch für so beachtlich gehalten, dass sich mit der Weblogforschung ein eigener Forschungszweig herausgebildet hat, der sich mit der Einbettung von Weblogs in den politischen Prozess befasst. Dem Blogging wird dabei, über die Blogger-Szene hinaus, Bedeutung als politischer Akteur zuerkannt. Mit dieser Kennzeichnung wird die Blogosphäre "zu einer relevanten politischen Teilöffentlichkeit bzw. zu einem festen Bestandteil der massenmedialen Öffentlichkeit« (Coenen 2005). Positiv gewertet werden insbesondere die Tendenzen des partizipatorischen Journalismus. Blogs werden damit zu Werkzeugen der politischen Kommunikation, die auf eine Verbesserung der »politischen Selbstverständigung der Bürgerschaft « und auf ein Gegengewicht zum kommerziellen System der Massenmedien mit seiner Meinungsmacht hoffen lassen (Coenen 2005).

Die einfachen Weblogs und die recht komplexen Diskurs-Plattformen repräsentieren ganz unterschiedliche Ausprägungen elektronischer Demokratie. Worin diese Unterschiede bestehen, möchte dieser Artikel in einem Rekurs auf die grundlegenden Ideen und Gestaltungsoptionen des Internets verdeutlichen. Mit Blick auf die Anwendungen und Praktiken des Web 2.0 soll dabei der Frage nachgegangen werden, ob sich in den grundlegenden Designprinzipien des Internets nicht nur die entscheidenden Hinweise auf die demokratischen Potenziale des Internets verbergen, sondern darüber hinaus auch Hinweise auf innovative Wege in der konkreten Ausgestaltung elektronischer Demokratie.

\section{Das demokratische Potenzial des Cyberspace}

Um zu verstehen, worin die demokratischen Potenziale des Internets bzw. die besondere demokratietheoretische Fazination des Internets liegt, lohnt ein Blick auf die Grundideen, die sowohl die Architektur als auch das technische Selbstreglement des Internets bestimmt haben. Faszinierend ist dabei besonders die Zeit des technischen Regimes, also jene Zeit, in der die technische Entwicklung und Verwaltung der Infrastruktur des Internets noch in den Händen einer informellen Gruppe akademisch geprägter Techniker lag. Im Mittelpunkt des Interesses steht das Internet in seiner Wahrnehmung als Cyberspace, d. h. als Struktur, die sich als ein Kommunikations- und Interaktionsraum begreifen lässt, der außerhalb der Ordnungslogik staatlicher Institutionen und des internationalen Staatensystems liegt. Im virtuellen Raum, der als Sphäre reinen Geistes keine physische Körperlichkeit kennt, spielen abgrenzbare Herrschaftsverbände mit territorialen Grenzen und einem staatlichen Gewaltmonopol keine Rolle. Die Besonderheit des Cyberspace liegt damit in der Abwesenheit von staatlicher Herrschaft (Bendrath 2007, S. 30). Diese be- 
sondere Unabhängigkeit weist auf den Bruch mit klassischen Macht- und Entscheidungsstrukturen hin, die sich bereits in der Um- und Durchsetzung technischer Standards und Gestaltungsprinzipien zeigen. Dave Clark vom Internet Architecture Board brachte diese Haltung mit zwei Sätzen auf den Punkt: »We reject kings, presidents, and voting. We belief in rough consensus and running code. « Die Sicherung der technischen Grundlagen des Internets erfolgt zwar durch internationale Organisationen wie die Internet Engineering Task Force (IETF), die Internet Numbers Authority (IANA) oder das W3C, im Unterschied zu traditionellen Regierungen und Standardisierungsgremien setzen diese Einrichtungen jedoch auf offene Standards, denen freiwillig gefolgt wird. Die Legitimation dieser eher informellen Organisationen ohne Rechtsstatus beruht dabei im Wesentlichen auf der technischen Expertise ihrer Mitglieder und der Transparenz der Entscheidungsvorgänge. Das Besondere dabei: Die Entscheidungen und verabschiedeten Standards der Internet-Organisationen konnten sich nur durchsetzen, weil deren Nutzen erkannt und der Organisation vertraut wurde. Mit dem Fehlen jeglicher Sanktionsmittel beruht die technische Organisation des Internets somit auf der Fähigkeit, einen Konsens über technische Standards und Regeln herzustellen. Die Standards und Regeln sind dabei im Netz selbst entstanden, ohne aktive Einmischung der Politik und ohne eine zentrale Instanz (Ahlert 1996). Zu den wichtigsten Besonderheiten dieser Art des Selbstreglements zählen Offenheit, Einfacheit, Inklusion, Bottom Up, Konsensorientierung und Dezentralität. Alle Betroffenen dürfen an der Ausgestaltung des Netzes mitwirken und nur den im Konsens getroffenen Entscheidungen wurde eine reelle Chance auf Durchsetzbarkeit eingeräumt (Hoffmann 2005, S. 10). Die Prinzipien dieser Art der Self-Governance finden in der technischen Architektur des Internets ihre Entsprechung: So liegt dem Internet ein radikal dezentralisiertes und minimalistisches Netzwerkkonzept zugrunde, das im Kern nur aus einer Sammlung offener, rechtlich nicht geschützter Protokolle besteht, die festlegen, wie Computer miteinander kommunizieren (Hoffmann 2005, S. 5). Charakteristisch ist das end-toend Prinzip, d. h. eine Richtlinie die festlegt, dass ein Netzwerk nur für die Übermittlung von Daten zu sorgen habe, sodass an deren Enden eine beliebige Anwendung laufen kann. Die Internetprotokolle konstituieren damit ein Netz autonomer Netze ohne zentrale Steuerung, das die Kontrolle des Datenflusses an die Endnutzer delegiert (Hoffmann 2005; Sandvig 2003).
Diese dezentrale Netzwerkstruktur bildet die technische Grundlage für die kommunikative Vernetzung von Menschen in einer polyzentrischen, netzwerkartigen Struktur. Das demokratische Potenzial des Internets hat damit seine Grundlage in der horizontalen und offenen Netzwerkarchitektur. Howard Rheingold hat die darauf gedeihende Kommunikationsstruktur als many-to-many-Kommunikation bezeichnet, $\mathrm{d}$. h. als Kommunikation von jedem mit jedem in virtuellen Communitys (Rheingold 1993). Es ist gleichsam die Grundlage, die das Internet als ein modernes und effizientes Kommunikationsmedium auszeichnet. Die Effizienz der Online-Kommunikation macht das Publizieren nicht nur billiger und schneller, sondern auch demokratischer, weil es prinzipiell jedem ermöglicht zu publizieren. Wird das Internet als modernes Massenmedium begriffen, ist in dieser Struktur verheißungsvoll verwirklicht, was bereits Berthold Brecht in seiner Radiotheorie forderte: Die Demokratisierung des Rundfunks durch seinen Wandel vom Distributions- zum Kommunikationsapperat, d. h. dem Vermögen, „den Zuhörer nicht nur hören, sondern auch sprechen zu machen und ihn nicht zu isolieren, sondern ihn in Beziehung zu setzen.«[5]

Es ist vor allem diese Besonderheit des Internets in seiner Eigenheit als ein sich selbst regulierender Raum, der den Visionen und Mutmaßungen über die Potenziale des Internets als ein Organ der »Demokratisierung von unten« den Grundstoff liefert und dabei auch die Frage aufwirft, ob die technische Selbstregulation des Internets Modell künftiger demokratischer Beteiligungsformen sein kann. Welcher Ort scheint geeigneter als ein in diesem Sinne herrschaftsfreier Raum für Jürgen Habermas Vision einer diskursiven Öffentlichkeit und dessen Zentralelement, dem rationalen, herrschaftsfreien Diskurs?[6] Vor diesem Hintergrund verwundert es wenig, dass sich mit der Entwicklung der ersten Computernetzwerke auch die Idee der Cyberdemokratie herausbildete. Diese in den 80er Jahren in den Vereinigten Staaten geborene Idee wurde von der Hoffnung getragen, dass durch die Bildung virtueller Gemeinschaften die Grundlage für einen demokratischen Aufbau der Gesellschaft gelegt werden könne. Das Modell war die elektronische Agora als Ort nicht-hierarchischer und freier Webkommunikation unter Freien und Gleichen. Ähnlich wie im Selbstreglement der technischen Architekturen des Internets dominiert auch hier die Ablehnung zentralistischer Regierungsformen und die Vorliebe für Konzepte der Selbstorganisation (Barbrook und Cameron 1997). 


\section{Das demokratische Potenzial des Web 2.0}

Die vielbeschworene many-to-many-Struktur ist auf der Ebene des Internets als Programmierplattform nichts anderes als ein Potenzial möglicher many-to-many-Vernetzungen. Die konkrete Umsetzung der Kommunikationsstruktur manifestiert sich erst in den Webanwendungen. Erst auf dieser Ebene zeigt sich die konkrete Ausformung von Geschäftsprozessen oder Sozialpraktiken. So lassen sich die Grundprinzipien der Selbstregulation aus den frühen Tagen des Internets, wie Offenheit, Einfachheit, Bottom Up, Nutzerpartizipation, Konsensorientierung und Dezentralität wohl in der netzwerkartigen Kommunikationsstruktur der Blogosphäre finden, jedoch weniger auf den monolithischen, top-down moderierten Diskursplattformen.

In der Beschaffenheit der Kommunikationsstruktur liegt der wesentliche Unterschied zwischen den Anwendungen des Web 1.0 und des Web 2.0. Die Bezeichnung Web 2.0 [7] wird mit einer ganzen Reihe technologischer und sozialer Trends verbunden, die in den letzten Jahren zu einer neuen Dynamik des Internets geführt haben. Dazu zählen zum einen neue Softwaretypen wie Blogs und Wikis, aber auch Visualisierungs-, Erschließungs- und Vernetzungstechniken von Webinhalten, wie Social Tagging, Recommendation-Systeme oder RSS-Feeds. Zum anderen bezieht sich dieser Begriff auf die Beobachtung einer sich grundlegend verändernden Nutzungspraxis des Internets, die insbesondere durch das Auftauchen neuer Akteurs- und Organisationstypen gekennzeichnet ist. Das Web 2.0 steht damit auch für den aktiven Internetnutzer, der als Prosument (Toffler 1989) oder professioneller Amateur (Leadbeater und Miller 2004) in selbstorganisierten Communitys zur Unterhaltung, Information, Aufklärung, Produktentwicklung und Meinungsbildung beiträgt. Obwohl das Web 2.0 durchaus auch für die technologische Weiterentwicklung des Internets steht, bezieht sich dieser Begriff weniger auf eine spezifische Technologie oder Innovation, sondern auf die konsequente Nutzung bereits bekannter Technologien und eines veränderten Nutzungsverständnisses. Das Web 2.0 ist in diesem Sinne, wie es der Blogger Ian Davis recht treffend formuliert hat, eine Haltung und keine Technologie [8].

Der Schlüssel zum Verständnis dieses Phänomens liegt im Wandel des Menschenbildes verborgen, in der Veränderung der Wahrnehmung, die Internetnutzer nicht nur als amorphe und tendenziell inkompetente
Masse erscheinen lässt, sondern als intelligentes Potenzial, das erst durch den konsequenten Einsatz von Kommunikationstechnologien zur vollen Blüte gelangen kann. Howard Rheingold spricht von Smart Mobs (Rheingold 2002), James Surowiecki von der Weisheit der Vielen/The Wisdom of Crowds (Surowiecki 2007) und Tim O'Reilly von kollektiver Intelligenz (O'Reilly 2005). Vor dem Hintergrund dieser Visionen eines Zeitalters vernetzter Intelligenz wird deutlich, dass sich hinter dem Begriff Web 2.0 im Kern die Durchsetzung einer Sozialpraxis verbirgt, die auf offener und dezentraler Kooperation beruht. Diese Praxis, die im Vertrauen auf soziale Netzwerkeffekte und damit auf kollektive Intelligenz setzt, lässt sich als Bruch mit der Tradition formal rationaler Organisation deuten. Wie radikal dieser Bruch ist, wird deutlich, wenn die man die Visionen und Ideen des Web 2.0 mit dem Ideal bürokratischer Organisation kontrastiert. Der Soziologe Max Weber hat die Leistungsfähigkeit bürokratischer Organisation noch idealtypisch als maschinenhaften bzw. regelgeleiteten Mechanismus beschrieben (Weber 1976):

»Ein voll entwickelter bürokratischer Mechanismus verhält sich zu diesem genau wie die Maschine zu den nicht mechanischen Arten der Gütererzeugung. Präzision, Schnelligkeit, Eindeutigkeit, Aktenkundigkeit, Kontinuierlichkeit, Diskretion, Einheitlichkeit, straffe Unterordnung, Ersparnisse an Reibungen, sachlichen und persönlichen Kosten und bei streng bürokratischer, speziell: monokratischer Verwaltung durch geschulte Einzelbeamte gegenüber allen kollegialen oder ehren- und nebenamtlicher Formen auf das Optimum gesteigert. Sofern es sich um komplizierte Aufgaben handelt, ist bezahlte bürokratische Arbeit nicht nur präziser, sondern im Ergebnis oft sogar billiger als die formell unentgeltliche ehrenamtliche.«

In der hier beschriebenen Regelhaftighaftigkeit bürokratischer Verwaltung lokalisiert Weber deren herausragende technische Überlegenheit und den entscheidenden Grund für den Siegeszug der Bürokratie. Webers Beschreibung enthält, was vielen immer noch als Garant effizienter Organisation gilt: hierarchische Ordnung, mechanische Präzision, Eindeutigkeit, Vermeidung von Reibungsverlusten, straffe Unterordnung, professionelle Tätigkeit. Das Web 2.0 lässt sich als Gegenentwurf zum Verständnis formaler Rationalität, hierarchischer Organisation und professionalisierter Arbeit begreifen: An die Stelle zentralistischer und hierarchischer Organisationsstrukturen treten dezentrale 
und selbstorganisierte Zusammenhänge, aus dem hierarchischen Top-Down Modell wird das Netzwerk.

Mit Rückblick auf die Frühphase des Internets fehlte dem Web 1.0 nicht die freiheitlich gestaltete Basisarchitektur, sondern die partizipative Praxis eines selbstorganisierten »Mitmach-Webs«, die nicht nur die Welt technikversierter Visionäre erfasst, sondern auch den weniger technikversierten Menschen zugänglich wird. Das Web 2.0 erscheint dabei weniger als Marketingidee, sondern als Umsetzung der Urideen und Konzepte des Cyberspace auf die Ebene der Webanwendungen.

\section{Zur Einschätzung der demokratischen Potenziale des Internets}

Die von den Visionären eines Web 2.0 beschworene Kraft gemeinschaftlich entfalteter Vernunft, die sich auf der Grundlage moderner Kommunikationstechnologien herausbilden kann, ist keinesfalls unumstritten. Kritische Gegenpositionen zeigen sich in der Diskussion um die Digitale Spaltung der Gesellschaft und im generellen Zweifel an der Fähigkeit virtueller (Laien-) Gemeinschaften Sinnvolles zu produzieren.

Die Ideen und Ideale der Cyberdemokratie wurden bereits Ende der 1990er Jahre von den Soziologen Richard Barbrook und Andy Cameron als »Kalifornische Ideologie« kritisiert. Kritikwürdig erschien ihnen vor allem die Annahme, dass der Zugang zu Informationen automatisch zur Ablösung traditioneller demokratischer Institutionen und zur Verlagerung der politischen Willensbildung in den virtuellen Raum führen werde. Dieser Ideologie werfen sie einen naiven Technikdetermismus vor, der blind mache, für die anwachsende soziale Kluft zwischen den »Information Rich« und den »Information Poor«bzw. für die Gefahren einer »Digitalen Spaltung « der Gesellschaft (Barbrook und Cameron 1997). Die international geführte Diskussion um die Risiken der Digitalen Spaltung ist von der Sorge getragen, dass die Nutzung moderner Informations- und Kommunikationstechnologien soziale Ungleichheit und gesellschaftliche Konflikte nicht entschärft, sondern verschärfen könnte. Als Indizien dafür werden der ungleich verteilte Zugang zum Internet, gravierende Ungleichheiten in den kommunikativen Fähigkeiten sowie der Medien- und Technikkompetenz herangezogen.

Empirische Befunde belegen, dass die Nutzung des Internets immer noch von Faktoren wie Geschlecht, Alter, formale Bildung, Einkommen und Berufstätigkeit abhängig ist (Hoecker 2002, Eurostat 2005, TNS-Infratest 2007, Initiative D21 2006). Mit den Anwendungen des Web 2.0 ist dieser Trend keinesfalls aufgebrochen. Die Diskrepanz von aktiver und passiver Nutzung pflanzt sich in den nutzergenerierten Inhalten (user generated content) fort. Gerade in der Bereitschaft und Fähigkeit, eigene Inhalte in das Netz zu stellen, wird deutlich, dass der aktive Netznutzer auch im Web 2.0 eine Minderheit (9\%) darstellt. Die meisten Menschen, insbesondere die älteren, einkommensschwachen und formal weniger gebildeten, nutzen das Internet nach wie vor passiv (TNS-Infratest 2007). Empirische Studien zeigen auch, dass die Qualität der Beiträge in Online-Diskursen zu wünschen übrig lässt (Hoecker 2002, Fuchs 2006) und ein einfacher Streifzug durch das Mitmach-Web macht offensichtlich, dass hier nicht nur Gutes, sondern auch viel Banales, sachlich Falsches, Geklautes, Diskriminierendes, Pornografisches, Kriminelles und Rassistisches zu finden ist.

Die empirischen Studien zeigen in der Tat, dass die Potenziale der many-to-many-Kommunikation nicht alle Netznutzer gleichermaßen einschließt. Die Studien können aber nicht belegen, ob dieser Befund tatsächlich die Schlussfolgerung zulässt, dass moderne Kommunikationstechnologien zu einer Verschärfung gesellschaftlicher Konflikte führen. Sowohl in der These von der Digitalen Spaltung als auch in den empirischen Untersuchungen, die diese These zu untermauern scheinen, präsentiert sich eine sehr verkürzte Sicht auf die Gefahren und Potenziale des Internets. Wovon konkret die Rede ist, das Internet in seiner Gesamtheit, seine Basisarchitektur oder ein bestimmter Anwendungstyp bleibt zumeist schwammig. Die eingesetzte Technik erscheint dabei in der Regel als gegeben und vermeintlich neutral. Verkürzte Sichtweisen fallen aber auch in den Positionen der zumeist technikversierten Visionäre einer Cyberdemokratie auf, die zumeist die Dynamik des gesellschaftlichen Kontextes unterschätzen. Obwohl sich die Cyberdemokraten auf die gesellschaftlichen Auswirkungen des Cyberspace beziehen, erscheint die Gesellschaft nur als Außenwelt, die automatenhaft auf technische Entwicklungen reagiert. In der Techniksoziologie werden die Folgen verengter Sichtweisen als Risiko technik-deterministischer Fehlschlüsse und technokratischer Sichtweisen thematisiert (Schwark 2003, Degele 2002). Technikdeterministische Positionen folgen in ihrer optimistischen Version der Annahme, dass sich bei hinreichender Durchsetzung einer Technolo- 
gie die gewünschten Änderungen im sozialen Kontext quasi automatisch einstellen würden. Die Weiterentwicklung der Demokratie wird damit als Automatismus technischer Innovation begriffen (Schwark 2003).

Die Debatte um Segen und Fluch des Internets bekommt jedoch eine neue Wendung, wenn das $\mathrm{Zu}$ sammenwirken von technischen Architekturen und menschlichem Handeln herausgestellt wird. Der amerikanische Rechtsgelehrte Lawrence Lessig stellt mit seiner These, dass der Code das Gesetz des Cyberspace sei (Code is Law), einen Zusammenhang von rechtlichen Normen und technischen Architekturen her. Der Code des Cyberspace, so seine These, entfalte als Regulationsinstanz mit ähnlicher Kraft wie Recht, Markt oder soziale Normen verhaltenssteuernde Wirkung. Die Architektur des Internets lässt sich in diesem Sinne als eine von Menschen geschaffene, sozial wirksame Umwelt begreifen, in der die in Code gefassten Architekturentscheidungen darüber bestimmen, welche Nutzungsweisen das Internet bietet und welche Nutzungsweisen es ausschließt (Lessig 2001). Ähnlich argumentiert Reidenberg, der in Anlehnung an die Lex Mercatoria des Handelsrechts von der Lex Informatica spricht. Wie Lessig geht auch Reidenberg davon aus, dass Recht und Regulierungspraktiken nicht als einzige Quelle der Verregelung zu betrachten sind, sondern dass technische Architekturen wie Recht wirksam werden und bestimmte Handlungsweisen erzwingen oder ausschließen (Reidenberg 1998). Mit der Betonung der normsetzenden Kraft technischer Architekturen rückt zunächst ins Bewusstsein, dass Software, ebenso wie die Protokolle des Internets, sozialen Ursprungs sind. Sie sind nicht aus dem Nichts entstanden, sondern werden von Menschen gemacht. In ihrer Gemachtheit spiegelt Software immer auch die kulturellen Vorstellungen ihrer Auftraggeber und Entwickler. Das heißt, es sind immer auch die Rationalitätsvorstellungen, Stereotype und Handlungsnormen von Menschen, die in die Designentscheidungen von Software einfließen. Software ist in diesem Sinne sozial und nicht neutral (Krempl 2001).

Wenn technische Architekturen sozial sind und über normsetzende Kraft verfügen, bleibt die Frage nicht aus, inwieweit Software bzw. technische Architekturen auch politisch sind (Krempl 2001; Winner 2004). Sie lassen sich in diesem Sinne ähnlich wie Recht als Steuerungsinstrument einsetzen. Sie können somit immer auch bewusst geschaffenes Mittel der Verhaltenssteuerung sein und zur Verwirklichung sozialer Ziele dienen. Da- mit wird deutlich, dass sich die demokratischen Potenziale des Internets nicht stillschweigend als unumstößlich voraussetzen lassen. Der Ruf nach Regulation und Änderungen im Netzdesign, insbesondere der Versuch, das end-to-end Prinzip des Internets aufzugeben (Lutterbeck 2005), stellen somit immer auch das Internet als freie Infrastruktur in Frage. Damit stehen auch die demokratischen Potenziale des Internets zur Disposition, die jederzeit dem Sicherheitsdenken oder partiellen ökonomischen Interessen geopfert werden können.

\section{Fazit: Wer bestimmt die Form der E-Demokratie?}

Die Betonung der Bedeutung technischer Artefakte als Mittel der Steuerung und Interessendurchsetzung birgt allerdings auch das Risiko technik-deterministischer Fehlschlüsse, solange angenommen wird, dass bewusst gewählte Gestaltungsoptionen direkte Wirkung im Anwendungsfeld zeigen. Hier liegen auch die Verlockungen technokratischer Ansätze im Sinne eines technological fix, d. h. der Vorstellung, dass sich soziale Probleme technisch lösen lassen (Degele 2002, S.25; Etzioni und Remp 1972). Die Betonung der Gemachtheit, der normsetzenden Wirkung und der politischen Qualität technischer Architekturen eröffnet aber auch den Blick auf interessante Ansätze und Fragen, die in der Diskussion um die demokratischen Risiken und Potenziale des Internets ausgespart bleiben.

Wenn sich Sozialität in Webanwendungen zeigen kann, wenn in diese soziale Regeln eingeschrieben sind, die Einfluss darauf ausüben, wer mit wem auf welche Art und Weise kommunizieren kann, wenn sich Newsgroups, Chats, Diskussionsforen, moderierte Diskursplattformen, Wikis oder Blogs als mögliche, aber nicht zwingende Varianten der many-to-many-Struktur des Internets begreifen lassen, wirft das die grundsätzliche Frage auf, wer denn legitimerweise die Form demokratischer Praxis bestimmt. Wer legt fest, wer miteinander kommunizieren kann, wer oder was vernetzt wird und wie die internetvermittelte Demokratie konkret aussieht? Neben der offenen Frage, wer die Form der E-Demokratie bestimmen soll, bleibt vor allem offen, wie es denn zur Praxis elektronischer Demokratie kommen kann oder mit anderen Worten, was E-Demokratie überhaupt ist. Wer legt fest, in welche Richtung sich die E-Demokratie entwickeln wird, was sie einschließt und was sie ausschließt? Woher beziehen die Entwickler ihre 
Modelle für die »innovativen Software-Lösungen «, die im Feld elektronischer Demokratie eingesetzt werden? Falls diese Modelle - wie so häufig - in den bekannten Praktiken der Offline-Welt gesucht werden, greift die Kritik des Medienwissenschaftlers Alexander Roesler, der sich darüber verwundert, dass die »utopische Dimension neuer Technik « so häufig im Vergangenen gesucht wird (Roesler 1997, S. 172). Soll diese Suche nach neuen Lösungen und Praktiken jedoch in einer bestenfalls schemenhaft erkennbaren Zukunft zu suchen sein, ist die Anwendungsdomäne E-Demokratie vor allem dadurch gekennzeichnet, das künftige Entwicklungen noch weitestgehend im Dunkeln liegen. In Roeslers Kritik deutet sich bereits an, dass sich die Wirkung einer neuen Technologie auf die demokratische Praxis nicht im Vorwege abschätzen lässt, mehr noch, dass einer neuen Kommunikationstechnologie, wenn sie sich nicht nur rückwärtsgewandt gebärdet, schlichtweg die konkrete Praxis fehlt, die sich dann verteufeln oder bejubeln lässt. Vor diesem Hintergrund erscheint es nicht verwunderlich, dass sich in der Literatur immer wieder Hinweise auf das Fehlen konkreter Handlungsansätze und Erfahrungen in der Umsetzung elektronischer Demokratie finden (Friedrichs, Hart, und Schmidt 2002; Kollbeck, Fazlik, und Ewert 2003).

Damit deutet sich ein erweitertes Verständnis elektronischer Demokratie an: E-Demokratie wird in diesem Sinne nicht nur als demokratische Auseinandersetzung entlang bestimmter Inhalte und Themen begriffen, sondern thematisiert auch die Ausgestaltung der Beteiligungspraxis selbst. Damit würde ein grundsätzlich anderer Weg beschritten werden als in der Entwicklung moderierter Diskursplattformen, die bereits die Form der demokratischen Auseinandersetzung vorwegnehmen. Konsequenterweise müsste dann versucht werden, die zivilgesellschaftlichen Akteure in die konzeptionelle Gestaltung der durch Software unterstützten demokratischen Praxis hineinzunehmen. Die Frage nach der Zukunft elektronischer Demokratie wäre damit auch eine Frage der Softwareentwicklung. Wenn das Web 2.0 als Demokratisierung der Medieninhalte zu begreifen ist, geht es hier um die Demokratisierung der Softwareentwicklung.

\section{Anmerkungen}

[1] Diese Definition findet sich im »BMI - Lexikon der Innenpolitik« auf der Homepage des Bundesinnenministeriums: www.bmi.bund. de.

[2] Die bislang bekanntesten Systeme sind die von der Firma TuTech Innovation GmBH entwickelte DEMOS Plattform und die von der Firma Binary Objects GmbH entwickelte Discourse Machine.

[3] Zur Kritik an den öffentlich finanzierten Online-Diskursen vgl. auch Dowe 2007 und Märker 2007.

[4] Der Begriff Blogosphäre bezeichnet die Gesamtheit der miteinander vernetzten Blogs und deutet auf die Wahrnehmung, dass Blogs durch ihre Vernetzungen Communitys bilden bzw. eine soziales Netzwerk repräsentieren.

[5] Grundlage für Brechts Radiotheorie bieten die folgenden Schriften: Der Rundfunk als Kommunikationsapparat. Rede über die Funktion des Rundfunks (1932/33), Vorschläge für den Intendanten des Rundfunks (1928/29), der Aufsatz »Radio - eine vorsintflutliche Erfindung?« (1927/28) und das Hörstück »Der Flug der Lindberghs. Ein Radiolehrstück für Knaben und Mädchen« (1929).

[6] In diesem Diskurs, der alle betroffenen Interessen berücksichtigt und nur das rationale Argument zum Zuge kommen lässt, sieht Habermas das wirksamste Mittel gegen die Demokratiedefizite repräsentativer Demokratien (Habermas 1999).

[7] Der Begriff Web 2.0 tauchte erstmalig im Jahre 2004 als Name einer Konferenzreihe (Web 2.0 Conference) auf, die von Tim O'Reilly (O’Reilly Media Verlag) und Craig Cline (MediaLive) initiiert wurde.

[8] »Web 2.0 is an attitude not a technology.«http://iandavis.com/ blog/2005/07/talis-web-20-and-all-that

\section{Literatur}

Ahlert, Ch. (1996): The Party is over: Vom selbstregierenden Internet zum regierten Cyberspace. In: Telepolis, Heise Zeitschriften Verlag, 07.07.2000, www.heise.de/tp/r4/artikel/8/8340/1.html [zugegriffen: 20.10.2007]

Barbrook, R./Cameron, A. (1997): Die kalifornische Ideologie: Wiedergeburt der Moderne? www.heise.de/tp/r4/artikel/1/1007/1.html [zugegriffen: 31.08.2008].

Bendrath, R. (2007): Demokrtiemaschine Internet? Das Netz als Projektionsraum politischer Utopien - und was daraus wurde. FIfFKommunikation 03/07, S. 30-33.

Coenen, Ch. (2005): Weblogs als Mittel der Kommunikation zwischen Politik und Bürgern. Neue Chancen für E-Demokratie? In: Schmidt, J./Schönberger, K./Stegbauer, Ch. (Hrsg.), Erkundungen des Bloggens. Sozialwissenschaftliche Ansätze und Perspektiven der Weblogforschung. Sonderausgabe, kommunikation@gesellschaft, Jg. 6 .

Degele, Nina (2002): Einführung in die Techniksoziologie. München: UTB Wilhelm Fink Verlag.

Dowe, Ch. (2007): Legitimationsschwund in der Politik. In: Stiftung Mitarbeit (Hrsg.), E-Partizipation. Beteiligungsprojekte im Internet, Bonn, S. 194-213.

Etzioni, A./Remp, R. (1972). Technological »Shortcuts« to social change. In: Science 175, S. 31-38.

Eurostat (2005): Die digitale Kluft in Europa. Online-Publikation, epp. eurostat.cec.eu.int [zugegriffen: 25.10.2006] 
Friedrichs, S./Hart, Th./Schmidt, O. (2002): Balanced E-Government. Visionen und Prozesse zwischen Bürgernähe und Verwaltungsmodernisierung. In: Aus Politik und Zeitgeschichte B 39-40/2002.

Fuchs, Ch. (2006): ePartizipation Research: A Case Study on Political Online Debate in Austria. In: ICT\&S Research Paper Series No 1. Salzburg: ICT\&S Center. www.icts.sbg.ac.at/content. php?id=1380\&m_id=1011\&ch_id=1379. [zugegriffen: 20.10. 2007]

Habermas, J. (1999): Die Einbeziehung des Anderen. Studien zur politischen Theorie. Frankfurt a. M.: Suhrkamp, 1. Auflage.

Hoecker, B. (2002): Mehr Demokratie via Internet? Die Potenziale der digitalen Technik auf dem empirischen Prüfstand. Online-Publikation, www.bpb.de [zugegriffen: 14.07.2006].

Hoffmann, J. (2005): Internet Governance: Eine regulative Idee auf der Suche nach ihrem Gegenstand. In: Schuppert, F. (Hrsg.): Governance-Forschung - Vergewisserung über Stand und Entwicklungslinien, Schriften zur Governance-Forschung, Baden-Baden: Nomos Verlag.

Initiative D21, TNS Infratest (2006): (N)Onliner Atlas 2006. Eine Topographie des Digitalen Grabens durch Deutschland. Online Publikation, www.nonliner-atlas.de [zugegriffen: 04.11.2007].

Kollbeck, J./Fazlik, N./Ewert, B. (2003): E-Demokratie. Stand, Chancen und Risiken. In: Schulzki-Haddouti, Ch. (Hrsg.): Bürgerrechte im Netz. Bonn, S. 227-260.

Krempl, St. (2001): TP: Soziale Software schreiben. In: Telepolis, www. heise.de/tp/r4/artikel/4/4893/1.html [zugegriffen 18.07.2008].

Leadbeater, Ch./Miller, P. (2004): The Pro-Am Revolution. How enthusiasts are changing our economy and society. Demos Publications, www.demos.co.uk/publications/proameconomy [zugegriffen: 21.08.2007].

Lessig, L. (2001): Code und andere Gesetze des Cyberspace. Berlin (Origninalsausgabe New York, 1999).

Lutterbeck, B. (2005): Infrastrukturen der Allmende - Open Source, Innovation und die Zukunft des Internets. In: Lutterbeck, B./ Gehring, R. A./Bärwolff, M. (Hrsg.): Open Source Jahrbuch 2005, Berlin: Lehmanns Media, S. 329-346.

Märker, O. (2007): Evaluation von E-Partizipation - am Beispiel onlinemoderierter Verfahren. In: Stiftung Mitarbeit (Hrsg.), E-Partizipation - Beteiligungsprojekte im Internet, Bonn.

O'Reilly, T. (2005): What is Web 2.0: Design Patterns and Business Models for the Next Generation of Software. www.oreillynet.com/ pub/a/oreilly/tim/news/2005/09/30/what-is-web-20.html.

Reidenberg, J. (1998): Lex Informatica: The Formulation of Information Policy Rules Through Technology. In: Texas Law Review 76(3): 553-584. http://reidenberg.home.sprynet.com/lex_informatica. pdf [zugegriffen: 05.09.2008].

Rheingold, H. (2002): Smart Mobs. The Next Social Revolution. Cambridge: Perseus.

Rheingold, H. (1993): The Virtual Community: Homesteading on the Electronic Frontier. New York: HarperPerennial.

Roesler, A. (1997): Bequeme Einmischung. Internet und Öffentlichkeit. In: Münker, St./Roesler, A. (Hrsg.), Mythos Internet, Frankfurt a. M.: Suhrkamp, S. 171-192.

Sandvig, C. (2003): Shaping Infrastructure and Innovation on the Internet: The End-to-End Network that isn't it. In: Guston, D./ Sarewitz, D. (Eds.), Shaping Science and Technology Policy: The Next Generation of Research. Madison: University of Wisconsin Press. Draft version, available at www.spcomm.uiuc.edu $\backslash$ csandvig $\backslash$ research $\backslash$ Communication_Infrastructure_and_Innovation. pdf. [zugegriffen: 25.09.2006].

Schwark, S. (2003): Politische Macht und das Internet. In: Rogg, A. (Hrsg.), Wie das Internet die Politik verändert - Einsatzmöglichkeiten und Auswirkungen, Opladen, S. 97-103.
Surowiecki, J. (2007): Die Weisheit der Vielen - Warum Gruppen klüger sind als Einzelne. München: C. Bertelsmann Verlag, 1. Auflage.

TNS-Infratest (2007): User Generated Content. TNS-Infratest, Media Research.

Toffler, A. (1989): The Third Wave. The Classic Study of Tomorrow. Random House Inc.

Weber, M. (1976): Wirtschaft und Gesellschaft. Grundriss der verstehenden Soziologie. Tübingen.

Winner, Langdon (2004): Do Artifacts have Politics? In: The Wale and the Reactor. A Search for Limits in the Age of High Technology, Chicago: University of Chicago Press, S. 19-39.

\section{Autor}

Dipl. Soz. Maika Büschenfeldt

Technische Fachhochschule Wildau

Fachbereich Wirtschaft, Verwaltung und Recht

Tel. +493375 508-379

maika.bueschenfeldt@tfh-wildau.de 